TP Periodica Polytechnica Mechanical Engineering

\author{
62(3), pp. 233-240, 2018 \\ https://doi.org/10.3311/PPme.12176 \\ Creative Commons Attribution (i)
}

RESEARCH ARTICLE

\section{On the Onset of Double-diffusive Convection in a Couple Stress Nanofluid in a Porous Medium}

\author{
Gian C. Rana ${ }^{1 *}$, Ramesh Chand ${ }^{2}$
}

Received 03 March 2018; accepted after revision 15 May 2018

\begin{abstract}
Double-diffusive convection in a horizontal layer of nanofluid in a porous medium is studied. The couple-stress fluid model is considered to describe the rheological behavior of the nanofluid and for porous medium Darcy model is employed. The model applied for couple stress nanofluid incorporates the effect of Brownian motion and thermophoresis. We have assumed that the nanoparticle concentration flux is zero on the boundaries which neutralizes the possibility of oscillatory convection and only stationary convection occurs. The dispersion relation describing the effect of various parameters is derived by applying perturbation theory, normal mode analysis method and linear stability theory. The impact of various physical parameters, like the couple stress parameter, medium porosity, solutal Rayleigh Number, thermo-nanofluid Lewis number, thermo-solutal Lewis number, Soret parameter and Dufour parameter have been examined on the stationary convection. It is observed that the couple stress parameter, thermo-nanofluid Lewis number, thermo-solutal Lewis number, Soret parameter and Dufour parameter have stabilizing effects on the stationary convection whereas the solutal Rayleigh number and Dufour parameter have very small effect on the system.
\end{abstract}

\section{Keywords}

nanofluid, porous medium, double-diffusive, convection, couple stress, Brownian motion, thermophoresis

\footnotetext{
${ }^{1}$ Department of Mathematics,

NSCBM Government College,

Hamirpur - 177005, Himachal Pradesh, India

${ }^{2}$ Department of Mathematics,

Government College,

Sugh-Bhatoli - 176204, Himachal Pradesh, India

*Corresponding author, e-mail: drgcrana15@gmail.com
}

\section{Introduction}

The onset of double-diffusive convection in nanofluid finds applications in the fields of chemical science, food processing, engineering and nuclear industries, geophysics, bioengineering and cancer therapy, movement of biological fluid, oceanography [1-9]. The word nanofluid was first proposed by Choi and Eastman [10]. Nanofluids are colloidal suspension of nanosized particles range between 1 to $100 \mathrm{~nm}$ [11] in the base fluid like water, oil, ethylene glycol etc. Nanoparticles are of materials such as metallic oxides $\left(\mathrm{Al}_{2} \mathrm{O}_{3}, \mathrm{CuO}\right)$, nitride ceramics (AlN, SiN), carbide ceramics ( $\mathrm{SiC}, \mathrm{TiC})$, metals $(\mathrm{Cu}, \mathrm{Ag}, \mathrm{Au})$ etc. have been used for the preparation of nanofluids.

Due to the small dimensions, the suspended nanoparticles can behave like a base fluid molecule, which helps us to reduce problems like particle clogging, sedimentation etc. The combination of highly stable and highly conductivity of the suspended nanoparticles which make them highly preferable for making heat transfer fluids [12-13]. The stable suspensions of small quantities of nanoparticles and increased heat transfer properties of nanofluids will possibly help us to design lighter, high performance thermal management systems and cost-effective liquid cooling systems.

A review article of heat transfer in nanofluid has been studied by Das and Choi [14] and a book [15]. Buongiorno [16] proposed a mathematical model for nanofluid based on the effects of Brownian motion and thermophoresis of suspended nanoparticles after analyzing the effect of seven slips mechanism, he concluded that in the absence of turbulent eddies, Brownian diffusion and thermophoresis are the dominant slip mechanisms.

Stokes [17] proposed and postulated the theory of couple-stress fluid. The application of couple-stress fluid is to the study of the mechanism of lubrication of synovial joints, which has become the main objective of scientific research and found that the synovial fluid in human joints behaves like a couple-stress fluid [18]. Sharma and Thakur [19] studied the couple-stress fluid heated from below in hydro magnetics and found that couple stress parameter has stabilizing effect on the stationary convection. Shivakumara et al. [20] studied the couple stress fluid under rotation in electro hydrodynamics 
and found that the couple stress fluid under rotation becomes destabilizing in the presence of couple stress for all the boundary conditons considered.

A convective transport in nanofluid was studied by [21-37]. In this paper, more realistic boundary conditions are used, see [38-39]. We assume that there is no flux at the plate and the nanoparticle flux value adjust accordingly. There is a need of changing the scale of dimensionless parameters. The basic solution of nanoparticle volume fraction is changed. The oscillatory convection does not exist and only stationary convection occurs.

Keeping in view of various applications of couple stress and nanofluid as mentioned above, our main aim in the present paper is to study the effect of cross-diffusion and couple stress parameter on the onset of convection in a horizontal layer of nanofluid saturating a porous medium. This problem is of triple-diffusion type as it contains heat, the nanoparticles and the solute.

\section{Mathematical Model and Governing Equations}

We consider an infinite horizontal porous medium layer of thickness $d$, bounded by the planes $z=0$ and $z=d$ of a couple stress nanofluid as shown in Fig. 1. The gravity force $\boldsymbol{g}=(0,0,-g)$ is acted aligned in the $z$ direction of the layer which is heated and soluted from below. Let $T_{0}, C_{0}$ and $\varphi_{0}$ be the constant values of temperature, concentration and the volumetric fraction of nanoparticles at the lower boundary and $T_{1}, C_{1}$ and $\varphi_{1}$ be the constant values of temperature, concentration and the volumetric fraction of nanoparticles at the upper boundary. We know that keeping a constant volume fraction of nanoparticles at the horizontal boundaries will be almost impossible in a realistic situation.

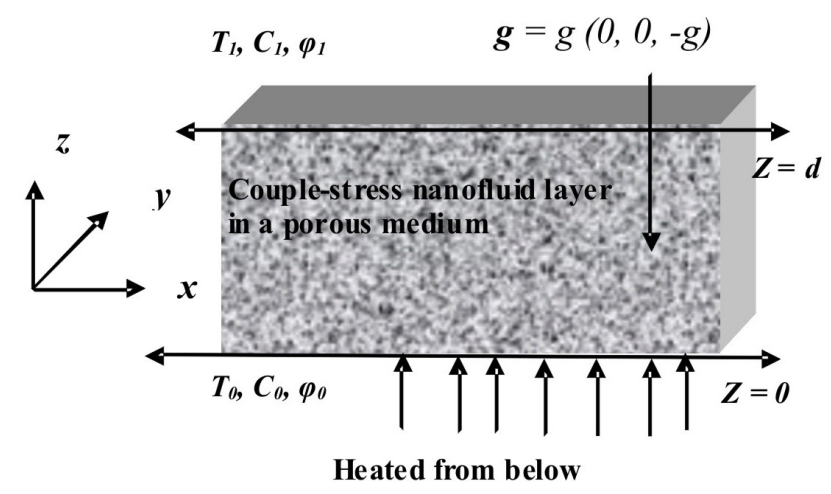

Fig. 1 Physical Configuration

Let $\rho_{0}, \varepsilon, \mu, \mu_{C}, p$ and $\boldsymbol{q}(u, v, w)$, denote respectively, the reference density, medium porosity, viscosity, the material constant responsible for couple stress property known as the couple stress viscosity, pressure, and Darcy velocity vector, respectively. $T$ is the temperature, $\varphi$ is the volume fraction of nanoparticles, $\rho_{p}$ is the density of nanoparticles, $\alpha_{T}$ is the coefficient of thermal expansion and $\alpha_{C}$ is analogous to solute concentration. The equations of continuity and motions for couple-stress nanofluid saturating a porous medium by applying Oberbeck-Boussinesq approximation [13, 17, 21, 22] are

$$
\nabla \cdot \boldsymbol{q}=0
$$

$$
\begin{aligned}
0 & =-\nabla p-\frac{1}{k_{1}}\left(\mu-\mu_{c} \nabla^{2}\right) \boldsymbol{q} \\
& +g\left(\varphi \rho_{p}+\rho_{0}(1-\varphi)\left\{\left(1-\alpha_{T}\left(T-T_{0}\right)-\alpha_{C}\left(C-C_{0}\right)\right)\right\}\right) .
\end{aligned}
$$

Let $D_{T}$ be the thermophoretic diffusion coefficient and $D_{B}$ is the Brownian diffusion coefficient. Assuming that the solute does not affect the transport of the nanoparticles. The equation of continuity $[16,24]$ for the nanoparticles in the presence of thermophoresis and absence of chemical reaction is

$$
\left(\frac{\partial}{\partial t}+\frac{1}{\varepsilon} \boldsymbol{q} \cdot \nabla\right) \phi=D_{B} \nabla^{2} \varphi+\frac{D_{T}}{T} \nabla^{2} T .
$$

The thermal energy equation for a nanofluid is

$$
\begin{aligned}
& (\rho c)_{m} \frac{\partial T}{\partial t}+(\rho c)_{f} \boldsymbol{q} \cdot \nabla T=k \nabla^{2} T+\varepsilon(\rho c)_{p} \\
& \left(D_{B} \nabla \varphi . \nabla T+\frac{D_{T}}{T} \nabla T . \nabla T\right)+\rho c D_{T C} \nabla^{2} C,
\end{aligned}
$$

where $c, c_{p}, k$ and $D_{T C}$ be the fluid specific (at constant pressure), the heat capacity of the material constituting nanoparticles, the thermal conductivity of the porous medium and the diffusivity of Dufour type, respectively.

Let $D_{S}$ and $D_{C T}$ be respectively, the solute diffusivity and diffusivity of Soret type and it is assumed that the nanoparticles do not affect the transport of the fluid. The conservation equation for solute concentration [24] is

$$
\left(\frac{\partial}{\partial t}+\frac{1}{\varepsilon} \boldsymbol{q} \cdot \nabla\right) C=D_{S} \nabla^{2} C+D_{C T} \nabla^{2} T .
$$

We assume that the temperature to be constant and the thermophoretic nanoparticles flux to be zero at the boundaries [27]. The appropriate boundary conditions $[38,39]$ are

$$
w=0, \frac{\partial w}{\partial z}=0, T=T_{0}, C=C_{0}, D_{B} \frac{\partial \phi}{\partial z}+\frac{D_{T}}{T_{1}} \frac{\partial T}{\partial z}=0 \text { at } z=0,
$$

$w=0, T=T_{1}, C=C_{1}, \frac{\partial w}{\partial z}=0, D_{B} \frac{\partial \phi}{\partial z}+\frac{D_{T}}{T_{1}} \frac{\partial T}{\partial z}=0$ at $z=d$.

Introducing non-dimensional variables as

$$
\begin{aligned}
& \left(x^{\prime}, y^{\prime}, z^{\prime},\right)=\left(\frac{x, y, z}{d}\right),\left(u^{\prime}, v^{\prime}, w^{\prime},\right)=\left(\frac{u, v, w}{\kappa_{f}}\right) d, \\
& t^{\prime}=\frac{t \kappa_{f}}{\sigma d^{2}}, p^{\prime}=\frac{p d^{2}}{\mu \kappa_{f}}, \varphi^{\prime}=\frac{\varphi-\varphi_{0}}{\phi_{1}-\phi_{0}}, T^{\prime}=\frac{T-T_{1}}{T_{0}-T_{1}}, \\
& C^{\prime}=\frac{C-C_{1}}{C_{0}-C_{1}},
\end{aligned}
$$


where $\kappa_{f}=\frac{k}{(\rho c)_{f}}$ is thermal diffusivity of the fluid and $\sigma=\frac{\left(\rho c_{p}\right)_{m}}{\left(\rho c_{p}\right)_{f}}$ is the thermal capacity ratio. Thereafter, dropping the dashes (') for convenience.

In non-dimensional form, Eqs. (1)-(8) can be written as

$$
\nabla . q=0
$$

$$
0=-\nabla p-\left(1-\eta \nabla^{2}\right) \boldsymbol{q}-R m \hat{e}_{z}+R a T \hat{e}_{z}+\frac{R s}{L e} C e_{z}-R n \varphi \hat{e}_{z},
$$

$$
\frac{1}{\sigma} \frac{\partial \varphi}{\partial t}+\frac{1}{\varepsilon} \boldsymbol{q} \cdot \nabla \varphi=\frac{1}{L n} \nabla^{2} \varphi+\frac{N_{A}}{L n} \nabla^{2} T
$$

$$
\frac{\partial T}{\partial t}+\boldsymbol{q} \cdot \nabla T=\nabla^{2} T+\frac{N_{B}}{L n} \nabla \varphi \cdot \nabla T+\frac{N_{A} N_{B}}{L n} \nabla T . \nabla T+N_{T C} \nabla^{2} C,
$$$$
\frac{1}{\sigma} \frac{\partial C}{\partial t}+\frac{1}{\varepsilon} \boldsymbol{q} \cdot \nabla C=\frac{1}{L e} \nabla^{2} \varphi+N_{C T} \nabla^{2} T
$$

$$
w=0, \frac{\partial w}{\partial z}=0, T=1, C=1, \frac{\partial \phi}{\partial z}+N_{A} \frac{\partial T}{\partial z}=0 \quad \text { at } z=0
$$

$w=0, \frac{\partial w}{\partial z}=0, T=0, C=0, \frac{\partial \phi}{\partial z}+N_{A} \frac{\partial T}{\partial z}=0 \quad$ at $z=1$

Where

$L e=\frac{\kappa_{f}}{D_{\mathrm{S}}} ;($ thermo-solutal Lewis number $)$

$\operatorname{Ln}=\frac{\kappa_{f}}{D_{B}} ;$ (thermo-nanofluid Lewis number)

$F=\frac{\mu_{c}}{\mu} ;$ (couple-stress parameter)

$R a=\frac{g \rho \alpha_{T} d k_{1}\left(T_{0}-T_{1}\right)}{\mu \kappa_{f}} ;$ (thermal Rayleigh number)

$R s=\frac{g \rho \alpha_{C} d k_{1}\left(C_{0}-C_{1}\right)}{\mu D_{S}} ;$ (solutal Rayleigh number)

$R m=\frac{\rho_{p} \varphi_{0}+\rho\left(1-\varphi_{0}\right) g d k_{1}}{\mu \kappa_{f}} ;$ (Density Rayleigh number)

$R n=\frac{\left(\rho_{p}-\rho\right) \phi_{0} g d k_{1}}{\mu \kappa_{f}} ;$ (nanoparticle Rayleigh number)

$N_{A}=\frac{D_{T}\left(T_{0}-T_{1}\right)}{D_{B} T_{1}\left(\phi_{1}-\phi_{0}\right)} ;$ (modified diffusivity ratio)

$N_{B}=\frac{(\rho c)_{p}}{(\rho c)_{f}}\left(\phi_{1}-\phi_{0}\right) ;($ modified particle-density ratio)

$N_{T C}=\frac{D_{T C}\left(C_{0}-C_{1}\right)}{\kappa_{f}\left(T_{0}-T_{1}\right)} ;$ (Dufour parameter)

$N_{C T}=\frac{D_{C T}\left(T_{0}-T_{1}\right)}{\kappa_{f}\left(C_{0}-C_{1}\right)} ;$ (Soret parameter).

\section{Perturbation Solutions}

To study the stability of the system, we superimposed infinitesimal perturbations on the basic state. We write

$$
\begin{aligned}
& \boldsymbol{q}(u, v, w)=q^{\prime \prime}(u, v, w), \quad T=T_{b}+T^{\prime \prime}, \\
& C=C_{b}+C^{\prime \prime}, \varphi=\phi_{b}+\varphi^{\prime \prime}, \quad p=p_{b}+p^{\prime \prime} .
\end{aligned}
$$

Using equations given in Eq. (16) into Eqs. (9)-(15), linearizing the resulting equations by neglecting nonlinear terms that are product of prime quantities and dropping the primes (") for convenience, we obtained

$$
\nabla . q=0
$$

$$
0=-\nabla p-\left(1-\eta \nabla^{2}\right) \boldsymbol{q}+R a T \hat{e}_{z}+\frac{R s}{L e} C \hat{e}_{z}-R n \varphi \hat{e}_{z}
$$

$$
\frac{1}{\sigma} \frac{\partial \varphi}{\partial t}+\frac{1}{\varepsilon} w=\frac{1}{L n} \nabla^{2} \varphi+\frac{N_{A}}{\operatorname{Ln}} \nabla^{2} T
$$

$$
\frac{\partial T}{\partial t}-w=\nabla^{2} T+\frac{N_{B}}{\operatorname{Ln}}\left(\frac{\partial T}{\partial z}-\frac{\partial \varphi}{\partial z}\right)-\frac{2 N_{A} N_{B}}{\operatorname{Ln}} \frac{\partial T}{\partial z}+N_{T C} \nabla^{2} C
$$

$$
\frac{1}{\sigma} \frac{\partial C}{\partial t}-\frac{1}{\varepsilon} w=\frac{1}{L e} \nabla^{2} C+N_{C T} \nabla^{2} T
$$

$w=0, T=0, C=0, \frac{\partial \phi}{\partial z}+N_{A} \frac{\partial T}{\partial z}=0$ at $z=0$ and at $z=1$.

Note that as the parameter $R m$ is not involved in Eqs. (17)-(21) it is just a measure of the basic static pressure gradient. In the absence of couple stress parameter and nanoparticles, the above system of Eqs. (17)-(21) reduced to the well-known equations for the double-diffusive Rayleigh-Bénard problem.

The seven unknowns $u, v, w, p, T, C$ and $\varphi$ can be reduced to four by operating Eq. (18) with $e_{z}$.curl curl, together with Eq. (17), which yields

$$
\left(1-\eta \nabla^{2}\right) \nabla^{2} w=R a \nabla_{H}^{2} T+\frac{R s}{L e} C \nabla_{H}^{2}-R n \nabla_{H}^{2} \varphi
$$

where $\nabla_{H}^{2}$ is the two-dimensional Laplace operator on the horizontal plane, that is $\nabla_{H}^{2}=\frac{\partial}{\partial x^{2}}+\frac{\partial}{\partial y^{2}}$.

The differential equations (23), (19)-(21) and the boundary conditions Eq. (22) constitute an eigenvalue problem which can be solved by the method of normal mode analysis method.

\section{Normal Modes Analysis Method}

We express the disturbances into normal modes of the form

$$
\begin{aligned}
& {[w, T, C, \varphi]=[W(z), \Theta(z), \Gamma(z), \Phi(z) \times]} \\
& \exp (i r x+i s y+p t),
\end{aligned}
$$

where $r, s$ are the wave numbers in the $x$ and $y$ direction, respectively, and $p$ is the growth rate of the disturbances.

Substituting Eq. (24) into Eqs. (23) and (19)-(22), we obtain the following eigenvalue problem

$$
\left(1-\eta\left(D^{2}-a^{2}\right)\right)\left(D^{2}-a^{2}\right) W+\omega^{2} R a \Theta+\frac{R s}{L e} \omega^{2} \Gamma-\omega^{2} R n \Phi=0
$$

$$
\frac{1}{\varepsilon} W+N_{C T}\left(D^{2}-\omega^{2}\right) \Theta+\frac{1}{L e}\left(D^{2}-\omega^{2}-\frac{p}{\sigma}\right) \Gamma=0,
$$




$$
\begin{gathered}
W+\left(D^{2}+\frac{N_{B}}{L n} D-\frac{2 N_{A} N_{B}}{L n} D-\omega^{2}-\frac{p}{\sigma}\right) \Theta \\
-\frac{N_{B}}{L n} D \Phi+N_{T C}\left(D^{2}-\omega^{2}\right) \Gamma=0 \\
\frac{1}{\varepsilon} W-\frac{N_{A}}{L n}\left(D^{2}-\omega^{2}\right) \Theta-\left(\frac{1}{L n}\left(D^{2}-\omega^{2}\right)-\frac{p}{\sigma}\right) \Phi=0,
\end{gathered}
$$

$w=0, T=0, C=0, D \phi+N_{A} D \Theta=0$ at $z=0$ and at $z=1$,

where $D=\frac{d}{d z}$ and $\omega^{2}=r^{2}+s^{2}$ is the dimensionless horizontal wave number.

Considering solutions $\mathrm{W}, \Theta, \Gamma$ and $\Phi$ of the form

$$
\begin{aligned}
& W=W_{0} \sin (\pi z), \Theta=\Theta_{0} \sin (\pi z), \Gamma=\Gamma_{0} \sin (\pi z), \\
& \Phi=\Phi_{0} \sin (\pi z) .
\end{aligned}
$$

Substituting Eq. (30) into Eqs. (25)-(28) and integrating each equation from $z=0$ to $z=1$, we obtain the following matrix equations

$$
\left[\begin{array}{cccc}
\left(1+\eta J^{2}\right) J^{4} & -\omega^{2} R a & -\frac{R s}{L e} \omega^{2} & \omega^{2} R n \\
\frac{-1}{\varepsilon} & J^{2} N_{C T} & \frac{J^{2}}{L e}+\frac{p}{\sigma L e} & 0 \\
-1 & J^{2}+p & -J^{2} N_{T C} & 0 \\
\frac{1}{\varepsilon} & \frac{N_{A}}{L n} J^{2} & 0 & \frac{J^{2}}{L n}+\frac{p}{\sigma}
\end{array}\right]\left[\begin{array}{c}
W_{0} \\
\Theta_{0} \\
\Gamma_{0} \\
\Phi_{0}
\end{array}\right]=\left[\begin{array}{l}
0 \\
0 \\
0 \\
0
\end{array}\right]
$$

where $J^{2}=\pi^{2}+a^{2}$ is the total wave number.

The linear system Eq. (31) has a non-trivial solution if and only if

$$
\begin{aligned}
R a & =\frac{1}{\varepsilon \sigma J^{2}+p \varepsilon+\sigma N_{T C} J^{2} L e} \times \\
& \left\{\begin{array}{l}
\frac{\varepsilon}{\omega^{2}}\left(1+\eta J^{2}\right) J^{4}\left[\left(J^{2}+p\right)\left(\sigma J^{2}+p\right)+L e N_{T C} N_{C T} J^{4}\right] \\
+R s \sigma\left[N_{C T} J^{2} \varepsilon-\left(J^{2}+p\right)\right]-\frac{R n \sigma}{J^{2}+p L n} \times \\
\left(J^{2} \sigma+p\right)\left(\begin{array}{l}
\left(\left(J^{2}+p\right) L n+\varepsilon N_{A} J^{2}\right)+ \\
\sigma N_{T C} J^{4} L e\left(N_{A}+L n N_{C T}\right)
\end{array}\right)
\end{array}\right\} .
\end{aligned}
$$

Equation (32) is the dispersion relation representing the effect of couple-stress parameter, medium porosity, thermosolutal Lewis number, thermo-nanofluid Lewis number, solutal Rayleigh Number, nanoparticle Rayleigh number, kinematic modified diffusivity ratio, Soret and Dufour parameteron double-diffusive convection in a layer of couple stress nanofluid saturating a porous medium.

\section{The Stationary Convection}

For stationary convection, we put $p=0$ in Eq. (32), reduces it to

$$
\begin{aligned}
R a & =\frac{1}{\varepsilon+N_{T C} L e} \times \\
& \left\{\begin{array}{l}
\frac{\varepsilon}{\omega^{2}}\left(\pi^{2}+\omega^{2}\right)^{3}\left(1+\eta\left(\pi^{2}+\omega^{2}\right)\right)\left(1+\operatorname{LeN}_{T C} N_{C T}\right) \\
+R S\left(N_{C T} \varepsilon-1\right)-R n\left[\begin{array}{l}
L n+\varepsilon N_{A} \\
+N_{T C} L e\left(N_{A}+L n N_{C T}\right)
\end{array}\right]
\end{array}\right\} .
\end{aligned}
$$

Equation (33) represents the thermal Rayleigh number as a function of the non-dimensional wave number $\omega$ corresponding to the parameters $\eta, \varepsilon, N_{T C}, N_{C T}, R s, L n, R n, L e, N_{A}$. Equation (33) is identical to that obtained by [24, 28, 29]. Also in Eq. (41) the particle increment parameter $N_{B}$ does not appear and the diffusivity ratio parameter $N_{A}$ appears only in association with the nanoparticle Rayleigh number $R n$. This implies that the nanofluid cross-diffusion terms approach to be dominated by the regular cross-diffusion term.

If we neglect the Dufour and Soret parameters $N_{T C}$ and $N_{C T}$, Eq. (33) reduces to

$$
\begin{aligned}
R a & =\frac{1}{\omega^{2}}\left(\pi^{2}+\omega^{2}\right)^{3}\left(1+\eta\left(\pi^{2}+\omega^{2}\right)\right)-\frac{1}{\varepsilon} R s \\
& -\frac{1}{\varepsilon}\left(L n+\varepsilon N_{A}\right) R n,
\end{aligned}
$$

which is identical with the result derived in [24, 28, 29]. In the absence of the solute gradient parameter $R s$, Eq. (33) reduces to

$$
\begin{aligned}
R a & =\frac{1}{\omega^{2}}\left(\pi^{2}+\omega^{2}\right)^{3}\left(1+F\left(\pi^{2}+\omega^{2}\right)\right) \\
& -\frac{1}{\varepsilon}\left(L n+\varepsilon N_{A}\right) R n,
\end{aligned}
$$

Equation (35) is identical with the results derived in [26-29]. Due to the absence of opposing buoyancy forces, the oscillatory convection does not exist. So we consider only the case of stationary convection.

\section{Results and Discussions}

To depict the stability characteristics, the dispersion relation Eq. (33) is analyzed numerically and graphs have been plotted. According to the definition of nanoparticle Rayleigh number $R n$, this corresponds to negative value of $R n$ for heavy nanoparticles $\left(\rho_{p}>\rho\right)$. In the following discussion, negative values of $R n$ are presented.

The variations of thermal Rayleigh number $R a$ with the wave number $\omega$ for different values of the couple stress parameter $\eta=0.2, \eta=0.4$ and $\eta=0.6$ is plotted in Fig. 2 and it is found that the thermal Rayleigh number $R a$ increases with the 


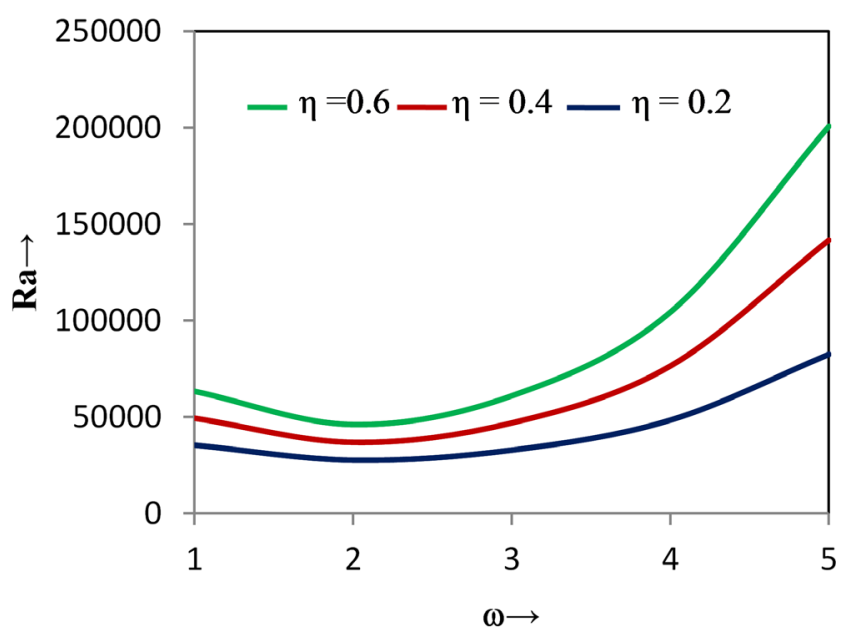

Fig. 2 The variations of Rayleigh number $R a$ with the wave number $\omega$ for different values of the couple stress parameter $\eta=0.2, \eta=0.4$ and $\eta=0.6$

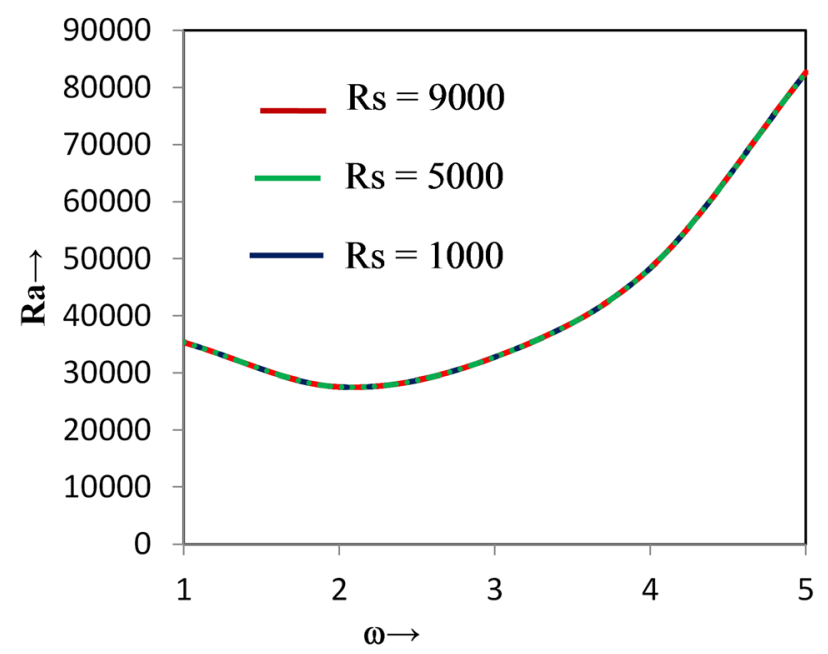

Fig. 3 The variations of Rayleigh number $R a$ with the wave number $\omega$ for different values of the solute concentration $R s=1000, R s=5000$ and $R s=9000$.

increase of couple stress parameter. Thus couple stress parameter stabilizes the stationary convection.

In Fig. 3, the variations of thermal Rayleigh number $R a$ with the wave number $\omega$ for three different values of the solutal Rayleigh number, namely, $R s=1000,5000$ and 9000 is plotted and it is noticed that the thermal Rayleigh number slightly increases with the increase in solutal Rayleigh number so the solutal Rayleigh number has very small effect on the stability of the system. In Fig. 4, the variations of thermal Rayleigh number Ra with the wave number $\omega$ for three different values of the thermo-nanofluid Lewis number, namely, $L n=300,600$ and 900 which shows that thermal Rayleigh number increases with the increase in thermo-nanofluid Lewis number. Thus thermo-nanofluid Lewis number has stabilizing effect on the system.

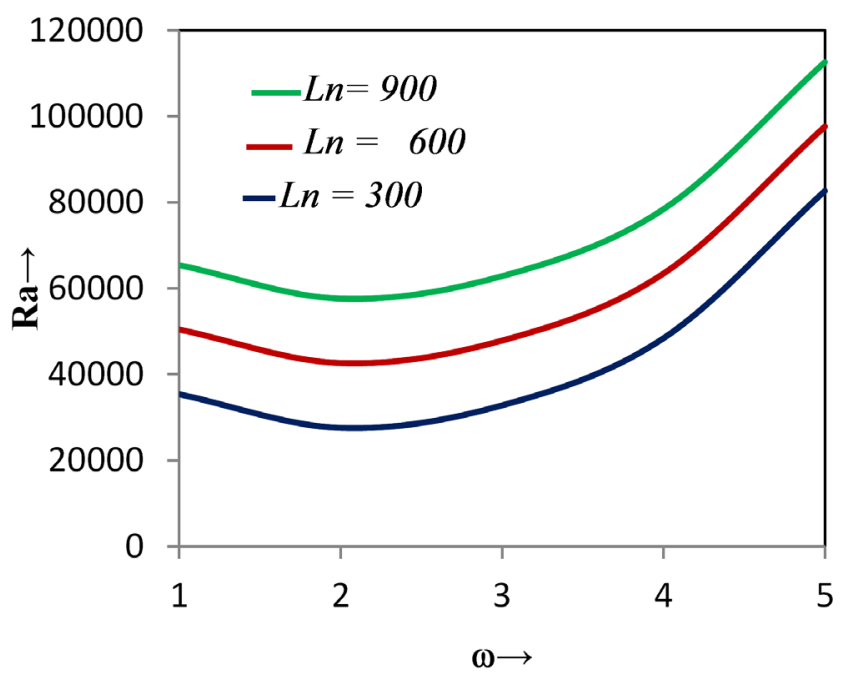

Fig. 4 The variations of Rayleigh number $R a$ with the wave number $\omega$ for different values of the thermo-nanofluid Lewis number $L n=300, L n=600$ and $L n=900$.

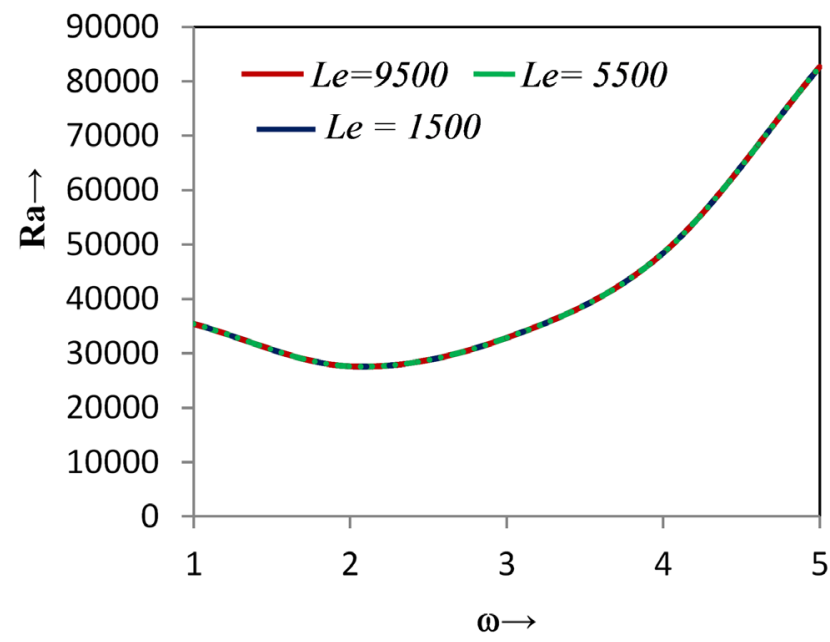

Fig. 5 The variations of Rayleigh number $R a$ with the wave number $\omega$ for different values of the Thermo-solutal Lewis number $L e=1500, L e=5500$ and $L e=9500$.

The variations of thermal Rayleigh number $R a$ with the wave number $\omega$ for three different values of the thermosolutal Lewis number, namely, $L e=1500,5500$ and 9000 is plotted in Fig. 5 and it is noticed that thermal Rayleighnumber increases slightly with the increase in thermosolutal Lewis number so the thermosolutal Lewis number has slightly stabilizing effect on the system.

In Fig. 6, the variations of thermal Rayleigh number $R a$ with the wave number $\omega$ for three different values of the Soret parameter, namely $N_{C T}=10,20,30$ which shows that thermal Rayleigh number increases with the increase in Soret parameter. Thus, Soret parameter has stabilizing effect on the system.

The variations of thermal Rayleigh number $R a$ with the wave number $\omega$ for three different values of Dufour parameter, namely $N_{T C}=5,45$ and 85 is plotted in Fig. 7 and it is observed 


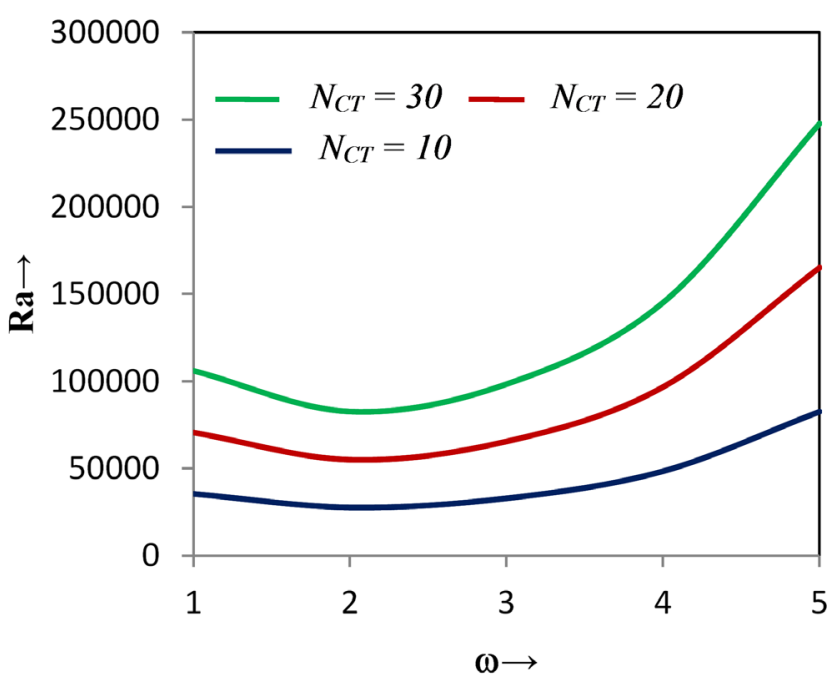

Fig. 6 The variations of Rayleigh number $R a$ with the wave number $\omega$ for different values of the Soret parameter $N_{C T}=10, N_{C T}=20$ and $N_{C T}=30$

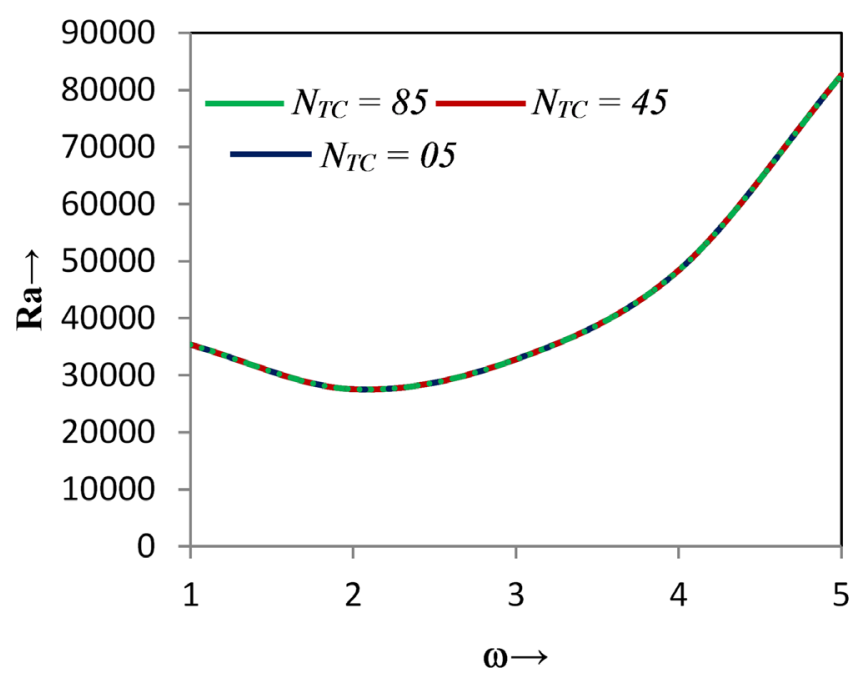

Fig. 7 The variations of Rayleigh number $R a$ with the wave number $\omega$ for different values of the Dufour parameter $N_{T C}=5, N_{C T}=45$ and $N_{C T}=85$

that thermal Rayleigh number increases with the increase in Dufour parameter so the Dufour parameter has stabilizing effect on the onset of stationary convection in a layer of couple-stress nanofluid. The system becomes more stable when the values of Soret and Dufour parameters are equal.

In Fig. 8, the variations of thermal Rayleigh number $R a$ with the wave number $\omega$ for three different values of the medium porosity, namely $\varepsilon=0.2,0.4,0.6$ which shows that thermal Rayleigh number increases with the increase in medium porosity. Thus medium porosity has stabilizing effect on the system.

The results obtained in Figs. 2 to 8 are in good agreement with the result obtained by [23-35].

\section{Conclusions}

The onset of double-diffusive convection in a porous medium layer of couple stress nanofluid in a more realistic boundary conditions has been investigated which comprises

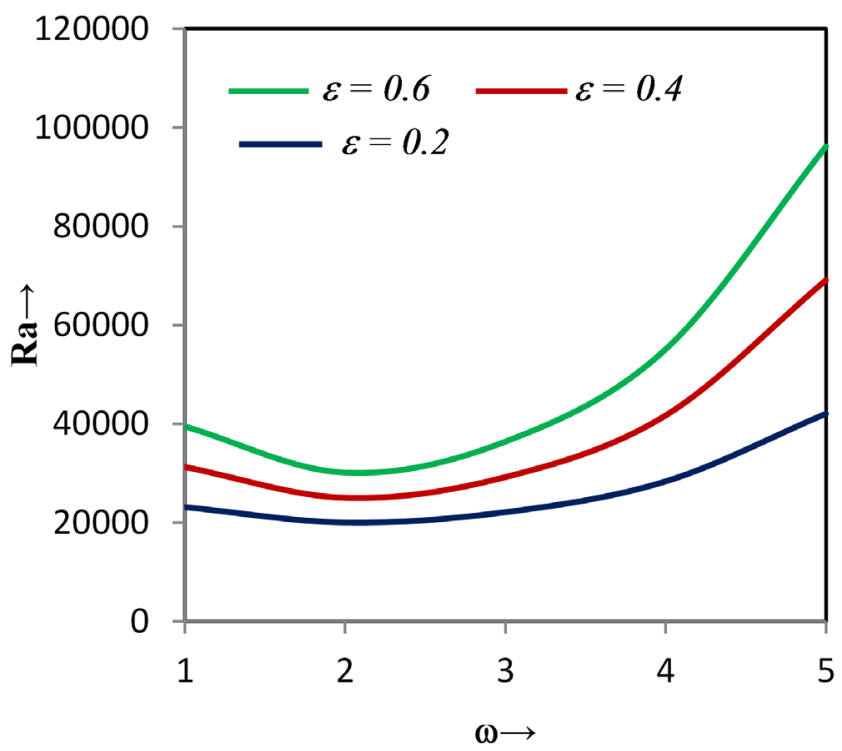

Fig. 8 The variations of Rayleigh number $R a$ with the wave number $\omega$ for different values of the medium porosity $\varepsilon=0.2, \varepsilon=0.4$ and $\varepsilon=0.6$.

the effects of thermophoresis and Brownian motion. It is found that the couple stress parameter has no effect on the basic solution. Nanofluid cross-diffusion terms approach to be dominated by the regular cross-diffusion term. The couple stress parameter, thermo-nanofluid Lewis number, thermo-solutal Lewis number, Soret parameter and Dufour parameter have stabilizing effects on the stationary convection as shown in Figs. 2, 4, 5, 6 and 8, respectively. The solutal Rayleigh number and Dufour parameter have very small effect on the system as can be seen in the Figs. 2 and 7, respectively. The system becomes more stable when the values of Soret and Dufour parameters are equal. Oscillatory convection does not exist under the more realistic boundary conditions.

\section{Acknowledgement}

Authors would like to thank the learned referee for his valuable comments and suggestions for the improvement of quality of the paper.

\section{References}

[1] Kim, S. Y., Koo, J.-M., Kuznetsov, A. V. "Effect of Anisotropy in Permeability and Effective Thermal Conductivity on Thermal Performance of an Aluminum Foam Heat Sink." Numerical Heat Transfer, Part A: Applications. 40(1), pp. 21-36. 2001.

https://doi.org/10.1080/10407780121436

[2] Kim, S. Y., Kuznetsov, A. V. "Optimization of Pin-fin Heat Sinks Using Anisotropic Local Thermal Nonequilibrium Porous Model in a Jet Impinging Channel." Numerical Heat Transfer, Part A: Applications. 44(8), pp. 771-787. 2003 https://doi.org/10.1080/716100528

[3] Boungiorno, J., Hu, L.-W. "Nanofluid coolants for advanced nuclear power plants." In: Proceedings of the American Nuclear Society - International Congress on Advances in Nuclear Power Plants 2005 (ICAPP'05), Seoul, South Korea, May 15-19, 2005. pp. 15-19. 
[4] Salloum, M., Ma, R. H., Weeks, D., Zhu, L. "Controlling nanoparticle delivery in magnetic nanoparticle hyperthermia for cancer treatment: Experimental study in agarose gel." International Journal of Hyperthermia. 24(4), pp. 337-345. 2008.

https://doi.org/10.1080/02656730801907937

[5] Salloum, M., Ma, R., Zhu, L. "An in-vivo experimental study of temperature elevations in animal tissue during magnetic nanoparticle hyperthermia." International Journal of Hyperthermia. 24(7), pp. 589-601. 2008. https://doi.org/10.1080/02656730802203377

[6] Salloum, M., Ma, R., Zhu, L. "Enhancement in treatment planning for magnetic nanoparticle hyperthermia: Optimization of heat absorption pattern." International Journal of Hyperthermia. 25(4), pp. 309-321. 2009. https://doi.org/10.1080/02656730902803118

[7] Kuznetsov, A. V., Nield, D. A. "The Onset of Double-Diffusive Nanofluid Convection in a Layer of a Saturated Porous Medium." Transport in Porous Media. 85(3), pp. 941-951. 2010 https://doi.org/10.1007/s11242-010-9600-1

[8] Nield, D. A., Kuznetsov, A. V. "The onset of double-diffusive convection in a nanofluid layer." International Journal of Heat and Fluid Flow. 32(4), pp. 771-776. 2011. https://doi.org/10.1016/j.ijheatfluidflow.2011.03.010

[9] Yadav, D., Lee, D., Cho, H.-H., Lee, J. "The Onset of Double-Diffusive Nanofluid Convection in a Rotating Porous Medium Layer with Thermal Conductivity and Viscosity Variation: A Revised Model." Journal of Porous Media. 19(1), pp. 31-46. 2016.

https://doi.org/10.1615/JPorMedia.v19.i1.30

[10] Choi, S. U. S., Eastman, J. A."Enhancing thermal conductivity of fluids with nanoparticles." In: International Mechanical Engineering Congress and Exhibition, San Francisco, USA, Nov. 12-17, 1995. [Online]. Available from: https://www.osti.gov/scitech/biblio/196525 [Accessed: 24th January 2018]

[11] Ganguly, S., Sikdar, S., Basu, S. "Experimental investigation of the effective electrical conductivity of aluminum oxide nanofluids." Powder Technology. 196(3), pp. 326-330. 2009. https://doi.org/10.1016/j.powtec.2009.08.010

[12] Choi, S. U. S., Zhang, Z. G., Yu, W., Lockwood, F. E., Grulke, E. A. "Anomolous thermal conductivity enhancement of nanotube suspensions." Applied Physics Letters. 79(14), pp. 2252-2254. 2001. https://doi.org/10.1063/1.1408272

[13] Eastman, J. A., Phillpot, S. R., Choi, S. U. S., Keblinski, P. "Thermal Transport in Nanofluids." Annual Review of Materials Research. 34, pp. 219-246. 2004. https://doi.org/10.1146/annurev.matsci.34.052803.090621

[14] Das, S. K., Choi, S. U. S. "A Review of Heat Transfer in Nanofluids." Advances in Heat Transfer. 41, pp. 81-197. 2009. https://doi.org/10.1016/S0065-2717(08)41002-X

[15] Das, S. K., Choi, S. U. S., Yu, W., Pradeep, T. "Nanofluids: Science and Technology." 1st ed., John Wiley \& Sons, Hoboken, New Jersey, USA, 2008.

[16] Buongiorno, J. "Convective Transport in Nanofluids." Journal of Heat Transfer. 128(3), pp. 240-250. 2006. https://doi.org/10.1115/1.2150834

[17] Stokes, V. K. "Couple Stresses in Fluids." Physics of Fluids. 9, pp. 17091715. 1966. https://doi.org/10.1063/1.1761925

[18] Walicki, E., Walicka, A. "Interia effect in the squeeze film of couple-stress fluids in biological bearings." Applied Mechanics and Engineering. 4(2), pp. 363-373. 1999. [Online]. Available from: https://www. infona.p1/resource/bwmeta1.element.baztech-article-BPZ2-0011-0021 [Accessed: 15th December 2012]
[19] Sharma, R. C., Thakur, K. D. "On couple-stress fluid heated from below in hydromagnetics." Czechoslovak Journal of Physics. 50(6), pp. 753758. 2000.

https://doi.org/10.1023/A:1022886903213

[20] Shivakumara, I. S., Akkanagamma, M.,Ng, C.-O. "Electrohydrodynamic instability of a rotating couple stress dielectric fluid layer." International Journal of Heat and Mass Transfer. 62, pp. 761-771. 2013. https://doi.org/10.1016/j.ijheatmasstransfer.2013.03.050

[21] Tzou, D. Y. "Thermal instability of nanofluids in natural convection." International Journal of Heat and MassTransfer. 51(11-12), pp. 29672979. 2008.

https://doi.org/10.1016/j.ijheatmasstransfer.2007.09.014

[22] Alloui, Z., Vasseur, P., Reggio, M. "Natural convection of nanofluids in a shallow cavity heated from below." International Journal of Thermal Sciences. 50(3), pp. 385-393. 2011.

https://doi.org/10.1016/j.ijthermalsci.2010.04.006

[23] Nield, D. A., Kuznetsov, A. V. "Thermal instability in a porous medium layer saturated by a nanofluid." International Journal of Heat Mass Transfer. 52(25-26), pp. 5796-5801. 2009.

https://doi.org/10.1016/j.ijheatmasstransfer.2009.07.023

[24] Yadav, D., Agrawal, G. S., Bhargava, R. "Thermal instability of rotating nanofluid layer." International Journal of Engineering Science. 49(11), pp. 1171-1184. 2011.

https://doi.org/10.1016/j.ijengsci.2011.07.002

[25] Yadav, D., Bhargava, R., Agrawal, G. S. "Numerical solution of a thermal instability problem in a rotating nanofluid layer." International Journal of Heat and Mass Transfer. 63, pp. 313-322. 2013.

https://doi.org/10.1016/j.ijheatmasstransfer.2013.04.003

[26] Sheu, L. J. "Thermal Instability in a Porous Medium Layer Saturated with a Viscoelastic Nanofluid." Transport in Porous Media. 88(3), pp. 461-477. 2011. https://doi.org/10.1007/s11242-011-9749-2

[27] Wang, S., Tan, W. "Stability analysis of soret-driven double-diffusive convection of Maxwell fluid in a porous medium." International Journal of Heat and Fluid Flow. 32(1), pp. 88-94. 2011. https://doi.org/10.1016/j.ijheatfluidflow.2010.10.005

[28] Chand, R., Rana, G. C. "Dufour and Soret Effects on the Thermosolutal Instability of Rivlin-Ericksen Elastico-Viscous Fluid in Porous Medium." Zeitschrift für Naturforschung A. 67(12), pp. 685-691. 2012. https://doi.org/10.5560/zna.2012-0074

[29] Rana, G. C., Thakur R. C., Kango, S. K. "On the Onset of Double-Diffusive Convection in a Layer of Nanofluid under Rotation Saturating a Porous Medium." Journal of Porous Media. 17(8), pp. 657-667. 2014. https://doi.org/10.1615/JPorMedia.v17.i8.10

[30] Yadav, D., Lee, J. "The onset of MHD nanofluid convection with Hall current effect." The European Physical Journal Plus. 130, pp. 162-184. 2015. https://doi.org/10.1140/epjp/i2015-15162-9

[31] Yadav, D., Lee, J. Cho, H. H. "Brinkman convection induced by purely internal heating in a rotating porous medium layer saturated by a nanofluid." Powder Technology. 286, pp. 592-601. 2015. https://doi.org/10.1016/j.powtec.2015.08.048

[32] Yadav, D., Nam, D., Lee, J. "The onset of transient Soret-driven MHD convection confined within a Hele-Shaw cell with nanoparticles suspension." Journal of the Taiwan Institute of Chemical Engineers. 58, pp. 235-244. 2016.

https://doi.org/10.1016/j.jtice.2015.07.008

[33] Yadav, D., Wang, J., Bhargava, R., Lee, J., Cho, H. H. "Numerical investigation of the effect of magnetic field on the onset of nanofluid convection." Applied Thermal Engineering. 103 pp. 1441-1449. 2016. https://doi.org/10.1016/j.applthermaleng.2016.05.039 
[34] Yadav, D. Mohamed, R. A., Cho, H. H., Lee, J. "Effect of Hall Current on the Onset of MHD Convection in a Porous Medium Layer Saturated by a Nanofluid." Journal of Applied Fluid Mechanics. 9(5), pp. 2379-2389. 2016.

https://doi.org/10.18869/acadpub.jafm.68.236.25048

[35] Yadav, D. "Electrohydrodynamic Instability in a Heat Generating Porous Layer Saturated by a Dielectric Nanofluid." Journal of Applied Fluid Mechanics. 10(3), pp. 763-776. 2017.

https://doi.org/10.18869/acadpub.jafm.73.240.27475

[36] Yadav, D., Mohamed, R. A., Lee, J., Cho, H. H. "Thermal convection in a Kuvshiniski viscoelastic nanofluid saturated porous layer." Ain Shams Engineering Journal. 8(4), pp. 613-621. 2017.

https://doi.org/10.1016/j.asej.2015.11.023
[37] Yadav, D., Wang, J. "Convective Heat Transport in a Heat Generating Porous Layer Saturated by a Non-Newtonian Nanofluid." Heat Transfer Engineering. 2018.

https://doi.org/10.1080/01457632.2018.1470298

[38] Nield, D. A., Kuznetsov, A. V. "Thermal instability in a porous medium layer saturated by a nanofluid: A revised model." International Journal of Heat Mass Transfer. 68, pp. 211-214. 2014.

https://doi.org/10.1016/j.ijheatmasstransfer.2013.09.026

[39] Yadav, D.,Agrawal, G. S., Lee, J. "Thermal instability in a rotating nanofluid layer: A revised model." Ain Shams Engineering Journal. 7(1), pp. 431-440. 2016.

https://doi.org/10.1016/j.asej.2015.05.005 